\title{
Evidence-Based PET for Neurological Diseases
}

\author{
Alberto Miceli, Selene Capitanio, \\ Maria Isabella Donegani, Stefano Raffa, \\ Anna Borra, Matteo Bauckneht, \\ and Silvia Morbelli
}

\subsection{Introduction}

Over the past two decades, one of the major breakthroughs for the approach to neurological diseases both in the clinical and research settings has been represented by the validation of diagnostic biomarkers able to demonstrate the presence of pathological mechanisms, alteration in neurotransmission as well as to predict disease progression $[1,2]$. The use of PET with different tracers as well as other imaging biomarkers support the etiological diagnosis of neurological disorders in vivo. This approach is particularly relevant in the field of neurodegenerative diseases. In fact, neurodegenerative diseases are characterized by the progressive degeneration and death of neurons. They represent a heterogeneous group of conditions characterized by different etiologies, different neuropathological and neurochemical alterations leading to different clinical pictures and courses [3]. Indeed, an early accurate diagnosis allows to tackle the disease with available or experimental intervention, lifestyle changes, or logistical arrangements, before disability has developed. Early intervention is

A. Miceli - S. Capitanio - M. I. Donegani - S. Raffa A. Borra $\cdot$ M. Bauckneht $\cdot$ S. Morbelli $(\bowtie)$

Nuclear Medicine Unit, IRCCS Ospedale Policlinico San Martino, Genoa, Italy

Department of Health Sciences, University of Genoa, Genoa, Italy

e-mail: silviadaniela.morbelli@hsanmartino.it expected to have greater clinical impact, extend independent and active life, improve its quality, and decrease the burden and costs of the disease [4]. However, the validation of PET tracers in neurological disease is still ongoing, and evidence on its comparative and combined diagnostic value with respect to other biomarkers is incomplete $[4,5]$. As a matter of fact, the increasing pressure for cost-effectiveness requires systematic assessment and validation of all biomarker performance in the clinical settings. Similarly only an evidence-based approach to new PET tracers can allow to select the most promising tracers for PET imaging in the research field both for pathophysiological investigations and for upcoming diagnostic approaches.

\subsection{Evidence-Based PET in Neurodegenerative Dementia}

Although the use of PET tracers for neurotransmission is also actively investigated, the vast majority of PET tracers recently developed for the clinical and pathophysiological evaluation of neurodegenerative dementia aim to evaluate the presence of specific pathological proteins deposition or mechanisms underlying neurodegeneration [3]. Tracers targeting neuroinflammation are also under investigation in this field but their use is still very far from the clinical 
setting [6]. Alzheimer's disease (AD) is by far the most relevant target for brain PET clinical imaging in neurodegenerative diseases [5]. The 2011 World Alzheimer Report has underlined that only a relatively small percentage of dementia cases (ranging between 20 and 50\%) are identified and correctly diagnosed in the early stages. This evidence means that at least half of the population of dementia patients does not receive a complete diagnostic workup at disease onset. This diagnostic delay gives rise to a socalled "treatment gap" between early stages of the disease and formal diagnosis, thus delaying correct care and preventing organized support which ameliorates patient's quality of life (and positively impacting also on caregivers and family members). In the field of $\mathrm{AD}$, two main categories of biomarkers have been proposed to identify the prodromal stage of disease [2, 7]. On the one side, amyloidosis biomarkers are able to identify the molecular/neuropathological feature of $\mathrm{AD}$ and include cerebrospinal fluid (CSF) amyloid- $\beta 1-42$ reduction and brain amyloid accumulation as imaged through PET technology using radiopharmaceuticals that selectively bind to the fibrillar aggregates of amyloid- $\beta$ plaques (AMY-PET) [2]. On the other side, neurodegeneration biomarkers reflect neuronal injury and downstream neurodegeneration, which can be measured by the increase of tau protein in the CSF, regional atrophy on MRI or synaptic metabolic dysfunction on fluorine-18 fluorodeoxyglucose PET $\left({ }^{18} \mathrm{~F}-\mathrm{FDG}\right.$ PET) [2]. As a matter of fact, the progressive dysfunction and loss of neurons lead to distinct involvement of functional systems and major clinical symptoms are mainly determined by the anatomical regions showing neuronal and synaptic dysfunction (which however do not necessarily reflect the molecular changes in the background) [8]. In this framework, MRI has both an exclusionary and inclusionary role for the early assessment of MCI. In fact on one side it can exclude secondary etiology of cognitive symptoms (i.e., vascular damage or normal pressure hydrocephalus) and it can increase the likelihood of a neurodegenerative dementia by highlighting the presence of atrophy in specific cortical regions [9]. Similarly, ${ }^{18}$ F-FDG PET is a well-founded method for evaluation of brain function and it is useful for the early diagnosis of $\mathrm{AD}$ and other dementias in people with mild cognitive impairment (MCI). ${ }^{18} \mathrm{~F}-\mathrm{FDG}$ PET is a sensitive and specific imaging modality available to support the etiological diagnosis of the underlying neurodegenerative dementia in demented patients. In particular, hypometabolism in the temporoparietal lobe, assessed by qualitative visual interpretation of the scans, represents the typical pattern found in AD [10]. However, despite its widespread use and the well-established role in the clinical settings, the quality of the available studies and thus the role of ${ }^{18} \mathrm{~F}$-FDG PET in identifying patients affected by AD who are still at the stage of MCI are less validated. As a matter of fact, discriminating between patients who will and will not progress to dementia due to AD is critical in the context of care and it must be pursued before patients have crossed the threshold into dementia. One of the background reasons associated with this surprising lack of evidence is related to evolving definition of MCI in the last decade. Unfortunately, the characteristics of the MCI patients recruited in the available published studies are quite diverse across the literature of the last 10 years and this heterogeneity is largely reflected but the lack of a reproducible value of sensitivity and specificity of ${ }^{18} \mathrm{~F}$-FDG PET for the identification of MCI due to AD. Moreover, the sensitivity and specificity of ${ }^{18} \mathrm{~F}$-FDG PET (as well as of all AD biomarkers) depend largely on the method of interpretation. It is, nowadays, advisable to use a combination of visual qualitative and semiquantitative analysis. In fact, the actual general sensitivity and specificity values, although still variable, are higher than $80 \%$ for both in the centers where it is utilized computer aided visual read approach such as Statistical Parametric Mapping (SPM), three-dimensional Stereotactic Surface Projection (3-D-SSP) statistics (Neurostat) or AD t-sum implemented in other software $[11,12]$. Software able to combine information from MRI and ${ }^{18} \mathrm{~F}$-FDG PET (even in the multicenter settings) have also been developed and have been demonstrated to 
improve detection and differentiation of $\mathrm{AD}$ and FTD (with $88-100 \%$ accuracy for AD detection) [13].

In more recent years, the availability of PET biomarkers of amyloid deposition such as ${ }^{11} \mathrm{C}$ $\mathrm{PiB}$ and ${ }^{18} \mathrm{~F}$-labeled tracers $\left({ }^{18} \mathrm{~F}\right.$-Flutemetamol, ${ }^{18} \mathrm{~F}$-Florbetaben, and ${ }^{18} \mathrm{~F}$-Florbetapir) has gained great attention [14]. These tracers exhibit high affinity binding for fibrillary amyloid that is a hallmark of $\mathrm{AD}$ and can be found in moderate to high number in cortical gray matter in all cases of $\mathrm{AD}$ developing many years before the onset of dementia. The vast majority of preliminary studies with amyloid PET has been carried out with ${ }^{11} \mathrm{C}$-PIB. It has been reported by measuring ${ }^{11} \mathrm{C}$ $\mathrm{PiB}$ distribution in $\mathrm{MCI}$ and control subjects, that MCI patients who converted to $\mathrm{AD}$, at an estimated rate of $25 \%$ per year, had greater ${ }^{11} \mathrm{C}-\mathrm{PiB}$ retention in several brain area with a $93.5 \%$ sensitivity and $56 \%$ specificity [15]. Moreover, none of the ${ }^{11} \mathrm{C}-\mathrm{PiB}$ negative $\mathrm{MCI}$ patients demonstrated to be converted to $\mathrm{AD}$ indicating that ${ }^{11} \mathrm{C}$ PiB negativity had a $100 \%$ negative predictive value for progression to $\mathrm{AD}[7,16]$. However, due to the 20 -min half-life of ${ }^{11} \mathrm{C},{ }^{11} \mathrm{C}$-PiB can only be used in PET centers with on-site cyclotron and radiopharmacy facilities while ${ }^{18} \mathrm{~F}$ is a more suitable radioisotope for widespread clinical use. Accordingly, the availability of fluorinated amyloid PET tracers has favored a greater impact of this tool also in the clinical settings [17]. In particular, a sensitivity of amyloid ${ }^{18} \mathrm{~F}$-labeled tracers ranging from 89 to $97 \%$, with a specificity ranging from 63 to $93 \%$ has been reported both for visual and semiquantitative analysis and no significant differences have been highlighted among the different agents [18].

The overall impacts of AMY-PET from the reported literature are a change of diagnosis and management in $35.2 \%$ and $59.6 \%$ of cases, respectively, leading to reduction in unnecessary investigations, treatments, relief of distress of caregivers, and potential involvement in clinical trials [19].

In particular, AMY-PET (as well as CSF data) are relevant for the differential diagnosis of etiologies underlying primary progressive aphasia (PPA). In fact, it has been suggested that the cur- rent clinical classification system for PPA should aim to predict underlying pathology across different cohorts and clinical settings which has a greater specificity with respect to neurodegeneration biomarkers (MRI and ${ }^{18}$ F-FDG PET) [20]. In fact, although patients' characteristics including age and ApoE genotype should be considered when interpreting AMY-PET, it has been highlighted that AMY-PET positivity is significantly more prevalent in logopenic variant of PPA, which belongs to the AD spectrum, (86\%) than in non-fluent variant of PPA $(20 \%)$ or semantic variant of PPA (16\%) which should be part of the spectrum of fronto-temporal degeneration [21]. It should be noted however that cognitive normal older people has a high burden of amyloid defined as incidental amyloidosis. Indeed, the presence of amyloid deposition is not sufficient to define the presence of pathophysiologic processes associated with $\mathrm{AD}$ which has to include the concomitant tau deposition [22]. Analyses in AMY-PET positive cognitive normal subjects have shown that the episodic memory and global cognitive function consistently had the largest, albeit still modest, effect sizes between performance and amyloid burden, whereas executive function, working memory, processing speed, visuospatial function, and semantic memory exhibited relatively smaller effect sizes [23]. Moreover, the incidence of brain amyloidosis increases with aging in all non-AD populations and has been repeatedly reported in other neurological conditions such as in dementia with Lewy bodies (DLB) and Parkinson's disease (PD). Similarly, all amyloid PET tracers have affinity to amyloid in vessel walls, and can thus result in positive scans in case of cerebral amyloid angiopathy (CAA) [7]. In this framework, it has been demonstrated that the ratio between the occipital and the whole brain uptake is higher in patients with CAA than in patients with $\mathrm{AD}$, possibly suggesting a more limited regional amyloid deposition on CAA [24]. Accordingly, AMY-PET has a moderate to good diagnostic accuracy for the identification of patients with CAA; especially as a negative scan it is useful to rule out CAA in the appropriate clinical setting [25]. Finally, it should be noted 
that soluble $\mathrm{A} \beta$ oligomers and neurofibrillary tangles (NFTs), the other histopathologic cores of Alzheimer's disease are not detected by this method, possibly producing false negative results [26].

\subsection{Evidence-Based PET for Movement Disorders}

Movement disorders are a group of neurologic syndromes characterized by an excess of movement or a scarcity of voluntary and automatic movements [27] related to different neurodegenerative or acquired central nervous system diseases affecting structures involved in movement control and modulation such as basal ganglia, cerebellum, cortex, and different thalamic nuclei. Parkinson's disease (PD) represents the second most common neurodegenerative disorder after $\mathrm{AD}$ and is typically characterized by motor and non-motor manifestations. Motor dysfunctions include bradykinesia, resting tremor, and muscular rigidity [28] as the result of dopaminergic deficit due to degeneration of the dopaminergic nigrostriatal system [29]. On the other hand, nonmotor features usually associated with the damage in non-dopaminergic pathways may include depression, olfactory and autonomic dysfunction, sleep disorders, psychiatric symptoms, pain, fatigue, and cognitive impairment [30] and can, in some case, even precede the motor phase by several years [31]. Besides the most prevalent PD, there are other related atypical parkinsonian syndromes (APS) also known as Parkinson-plus syndromes. From the neuropathological point of view, on the basis of the predominant multisystemic progressive accumulation of misfolded proteins, parkinsonian disorders could be classified into $\alpha$ synucleinopathies (PD with and without cognitive impairment/dementia, dementia with Lewy bodies-DLB, and multiple system atrophy-MSA) and tauopathies (corticobasal degeneration-CBD and progressive supranuclear palsy-PSP). All the aforementioned conditions must be distinguished from non-degenerative causes of parkinsonism (e.g., toxic/drug-induced, psychogenic, or vascular eti- ologies) in which presynaptic nigrostriatal pathways are preserved. Molecular imaging has proven to be a powerful tool for improving our understanding of the pathophysiology underlying parkinsonian disorders. In particular, PET and single photon emission computed tomography (SPECT) imaging are used as surrogate of unique in vivo functional measurement of monoaminergic pathways impairment in neurodegenerative diseases since the early 1980s [32]. Actually, these techniques are able to evaluate and detect nigrostriatal degeneration from different points of view. The analogue of levodopa ${ }^{18} \mathrm{~F}$-fluorodopa $\left({ }^{18} \mathrm{~F}\right.$-DOPA $)$ is a PET tracer that estimates the activity of aromatic amino acid decarboxylase enzyme (AADC), which converts levodopa into dopamine in striatal cells, thus reflecting dopamine synthesis. After its conversion in ${ }^{18}$ F-fluorodopamine, it follows the same fate of endogenous dopamine and is thus transported to intraneuronal storage vesicles by vesicular monoamine transporter 2 (VMAT2) [33] to then be released into the synaptic cleft and interact with postsynaptic dopamine receptors.

On the other hand, presynaptic dopamine transporter (DAT) density, responsible for reuptake of dopamine (DA) from the synaptic cleft and typically reduced in PD [34], can be measured through ${ }^{123}$ I-FP-CIT ( ${ }^{123}$ I-ioflupane), one of the most used radiotracers for SPECT imaging, or with specific PET tracers $\left({ }^{18} \mathrm{~F}-\mathrm{PE} 2 \mathrm{I}\right.$; $\mathrm{N}$-(3iodoprop-2Eenyl)-2 $\beta$-carbomethoxy-3 $\beta$-(4methyl-phenyl)nortropane). Finally, specific radiolabeled ligands for VMAT2 also exist and can be used in PET imaging for research purposes $\left({ }^{11} \mathrm{C}\right.$ - and ${ }^{18} \mathrm{~F}$-dihydrotetrabenazine). The applicability of functional imaging in the evaluation of striatal dopaminergic degeneration in control subjects and PD patients is well documented. Actually, PET and SPECT studies with AADC, VMAT2, and DAT tracers are able to monitor nigrostriatal deficit over time demonstrating the negative effects of age on DA transporters and receptors. According to Karrer et al. [35], age has a significantly larger effect on D1- than D2-like receptors with an average age reduction across the DA system of about 3.7-14.0\% per decade. On the contrary, DA synthesis capacity seems to 
be spared from this detrimental effect. This finding nicely fit with the results of a contemporary meta-analysis of Kaasinen et al. [36] including PD patients submitted to PET and SPECT studies for the evaluation of striatal presynaptic dopamine function. In this meta-analysis, it was demonstrated a higher defect in DAT and VMAT2 function compared to AADC defect in PD patients. This difference is possibly due to the effect of a compensatory upregulation in AADC function, confirming vesicular monoamine transporter 2 and dopamine transporter as the most sensitive targets to assess. In the same study, a linear correlation between disease severity and dopamine loss was demonstrated and a mean motor disease duration of 4-7 years was needed to overcome the general compensatory changes in the terminal area of the nigrostriatal tract in PD and induce a detectable loss of about $50 \%$ of putaminal presynaptic dopamine function. It should be noted, however, that ${ }^{18} \mathrm{~F}$-DOPA PET is a valid alternative to ${ }^{123}$ I-FP-CIT SPECT. Similarly, PET VMAT2 tracers as ${ }^{11} \mathrm{C}$ and ${ }^{18} \mathrm{~F}$-labeled dihydrotetrabenazine are very sensitive in detecting presynaptic dysfunction in PD without significant influence by compensatory changes [37] but are less available than other tracers. Besides dopaminergic dysfunction, serotonergic system exerts an important role in PD pathophysiology. Among PET ligands that have been developed for the evaluation of serotonergic receptors and serotonin transporter (SERT), ${ }^{11} \mathrm{C}$-DASB is a second-generation PET ligand with the best selectivity for the SERT [38]. In their systematic review examining the in vivo SERT changes in PD measured by ${ }^{11} \mathrm{C}$-DASB PET, Pagano et al. [39] demonstrated that SERT dysfunction is associated with the development of several motor (tremor and dyskinesias) and non-motor symptoms (depression, fatigue, apathy, and weight changes). In particular, they observe a highest decrease in SERT binding in the rostral raphe and caudate followed by putamen, thalamus, ventral striatum, caudal raphe, and hypothalamus, according to succession of pathological events in serotonergic neurons described by Braak's PD staging [29]. Furthermore, reduced SERT binding in putamen is associated with longer duration of the disease, whereas lower ${ }^{11} \mathrm{C}$-DASB binding in caudate is associated with worse cognitive function and older age. Interestingly, although SERT binding in putamen decreased with the progression of the disease, PD patients with dyskinesias have relatively preserved putaminal serotonergic function with mechanisms not fully clarified.

Moreover, PET and SPECT are able to detect pathologic changes from the earliest phase of the disease, improving diagnostic accuracy in the early stages [40]. REM sleep behavior disorder (RBD) confirmed by polysomnography is a parasomnia occurring during REM sleep characterized by the loss of physiological muscle atonia and associated with dream-enacted behaviors. It is defined "idiopathic" when appears isolated, without any other clinical sign of a neurological disorder. However, more than $80 \%$ of idiopathic RBD patients will develop a definite neurodegenerative disease, mostly a synucleinopathy. Furthermore, the presence of abnormal presynaptic dopaminergic PET or SPECT scan is considered the second most relevant risk factor for prodromal PD [41] predicting a high risk for short-term conversion into a synucleinopathy in idiopathic RBD patients [42]. A recent metaanalysis about the role of presynaptic dopaminergic imaging in RBD shows that idiopathic RBD patients exhibit decreased nigrostriatal dopaminergic functioning in comparison with healthy controls, especially at the putamen level. Furthermore, patients with idiopathic RBD and patients with PD without RBD exhibit a similar degree of nigro-caudate dopaminergic deafferentation [43].

Molecular imaging can also facilitate the differential diagnosis among PD, atypical parkinsonian syndromes (APS), essential tremors, and other degenerative conditions that represent a group of complex and heterogeneous diseases with overlapping symptomatology and variable response to dopaminergic medications. ${ }^{123} \mathrm{I}-\mathrm{FP}$ CIT SPECT can distinguish degenerative forms of parkinsonism from essential tremor [44], druginduced parkinsonism [45] and could also differentiate DLB from AD. Subjects with PD and APS show indeed an early reduction of striatal 
dopaminergic binding [46] usually before the appearance of motor symptoms. On the contrary, patients with essential tremor, drug-induced parkinsonism, and $\mathrm{AD}$ are characterized by normal dopamine transporter uptake [47]. Although a differential pattern has been described at the group level, a real distinction between the various degenerative forms of parkinsonism is not possible by means of SPECT assessment only. To this purpose, ${ }^{18} \mathrm{~F}-\mathrm{FDG}$ PET has demonstrated to be more promising. As suggested by Albrecht et al. [48], glucose hypometabolism at ${ }^{18} \mathrm{~F}$-FDG PET can identify consistent functional brain abnormalities in PD, outperforming structural MRI. In particular, while MRI showed only focal and inconsistent alterations, in PD patients the authors found an extensive glucose hypometabolism in bilateral inferior parietal cortex and left caudate nucleus that is related to cognitive deficits (inferior parietal cortex) and motor symptoms (caudate nucleus). Disease-specific patterns of regional glucose metabolism in patients with parkinsonism are well documented [49, 50]. However, the valuable capability of ${ }^{18} \mathrm{~F}-\mathrm{FDG}$ PET for accurate differentiation between PD and APS has been unanimously accepted only in recent years. In a preliminary meta-analysis, Meyer et al. well described the different ${ }^{18} \mathrm{~F}$-FDG uptake pattern in PD and APS [51]. PD is characterized by a posterior temporoparietal, occipital, and sometimes frontal hypometabolism with a relative hypermetabolism of the putamen, pallidum, thalamus sensorimotor cortex, pons, and cerebellum.

MSA patients show instead a marked hypometabolism of the putamen (mainly in its posterior portion), pons, and cerebellum, which may be more pronounced in the striatum or in the pons and cerebellum, on the basis of the clinical presentation. Conversely, PSP is characterized by a regional hypometabolism preferentially involving the medial, dorsal, and ventrolateral frontal areas (i.e., the anterior cingulate gyrus, supplementary motor area, precentral gyrus, and premotor-to-posterior prefrontal areas); caudate, thalamus, and upper brain stem. Finally, CBD patients have a typically highly asymmetric hypometabolism of the frontoparietal areas, striatum, and thalamus contralateral to the most affected body side. A concomitant cortical hypometabolism may be mainly found in the parietal cortex and usually extends across the sensorimotor cortex into the cingulate gyrus and premotorto-posterior prefrontal areas.

${ }^{18}$ F-FDG PET has proved to be particularly relevant also for the diagnosis of DLB and is actually listed among the supportive biomarkers for its identification [52]. DLB is characterized by a more prominent hypometabolism affecting the primary visual cortex and occipital cortex with relative preservation of subcortical structures and primary somatomotor cortex and with a concomitant hypometabolism in posterior associative cortex. In particular, the presence of a hypometabolism in the precuneus with a relative sparing of glucose uptake in posterior cingulate gyrus is known as "cingulate island sign" and has proved to significantly increase ${ }^{18} \mathrm{~F}-\mathrm{FDG}$ PET specificity for the differential diagnosis with respect to $\mathrm{AD}$, although lower than DAT SPECT [53].

${ }^{18} \mathrm{~F}$-FDG PET evidence is also available for Huntington's disease gene expression carriers (HDGECs). Actually, several PET imaging studies investigating the glucose metabolism in HDGECs have shown specific metabolic patterns mainly characterized by a progressive reduction of subcortical and cortical glucose metabolism in the striatum, thalamus, insula, posterior cingulate gyrus, and prefrontal and occipital cortex associated to a relative hypermetabolism in the cerebellum and pons [54]. In particular, reduction in striatal metabolism seems to be an early feature of the disease, preceding neuronal loss and thus motor onset of the disease. However, glucose metabolism deficits are only one of the many factors involved in Huntington's disease and ${ }^{18} \mathrm{~F}-\mathrm{FDG}$ PET is not indicated for the diagnosis of this disease. Besides ${ }^{18} \mathrm{~F}-\mathrm{FDG}$, other striatal PET radioligands have been used in this context. In particular, a meta-analysis aiming to investigate striatal molecular changes in 158 premanifest and 191 manifest HDGECs patients [10] demonstrates a significant decrease not only in glucose metabolism in caudate and putamen but also in dopamine D2 receptors and in striatal phosphodiesterase $10 \mathrm{~A}$ binding. This findings well reflect the different 
neuropathological mechanisms underlying the development of the disease.

Finally, PET and SPECT could guide the clinician in the choice of the different therapeutic modalities and in monitoring therapy response. PET has proved to be able to determine drug dosage for optimal efficacy in movement disorders as in the case of PD patients under deprenyl treatment evaluated with ${ }^{11} \mathrm{C}$-deprenyl PET [55]. ${ }^{18} \mathrm{~F}$-DOPA PET has been used in several studies to evaluate the effects of potential neuroprotective agents on dopaminergic function [56]. ${ }^{11} \mathrm{C}$-raclopride PET was used to evaluate striatal D2 receptor status in PD patients showing a normal or raised striatal D2 binding potential in untreated patients with PD but reduced in patients with $\mathrm{PD}$ and a fluctuating response to L-dopa [57].

Finally, PET and SPECT studies have proved the existence of a link between impulse control disorders (ICD) and dopamine activity dysfunction across ventral and dorsal striatum in PD patients [58]. ICD are a class of psychiatric disorders including pathological gambling, hypersexuality, binge-eating, and compulsive shopping that could appear in around $30 \%$ of PD patients as a complication of D2/3 dopamine agonist treatment and, to a lesser extent, levodopa. A recent meta-analysis demonstrated the PET/ SPECT dopaminergic striatal correlates of ICD in PD. In particular, in ICD patients authors find lower DAT levels in the dorsal striatum and in its subdivisions (i.e., putamen, caudate) and reduced binding (i.e., increased dopamine release) in the ventral striatum in response to reward-related stimuli or gambling task. These lines of evidence highlight the importance of PET pharmacokinetic and pharmacodynamic studies in our understanding of the mechanisms of action, efficacy, and complications of medical interventions in patients with neurological diseases.

\subsection{Evidence-Based PET for Psychiatric Disorders}

While in the clinical settings either ${ }^{18} \mathrm{~F}-\mathrm{FDG}$ or AMY-PET can be used to support the differential diagnosis between neurodegenerative dementia and depressive pseudodementia, in the specific field of psychiatric disorders, PET technology has more extensively been used for research purposes to determine pathophysiology of diseases and response to intervention [59]. In particular, the availability of tracers for imaging of neurotransmission allows the investigation of different systems (i.e., serotonin, dopamine GABA pathways) and to investigate a wide range of psychiatric diseases. Similarly, brain PET with different tracers (i.e., tracers for neuroinflammation) has been used to explore new hypotheses related to the onset of psychiatric diseases. The vast majority of PET studies carried out in the last 20 years in psychiatric patients have aimed to map functional alterations and mechanism underlying major depressive disorder (MDD), a common mental illness with high lifetime prevalence (close to 20\%.). Indeed, although the presence of aberrant brain activation during cognitive and emotional processing has been extensively evaluated in MDD patients, results of available studies vary considerably. Muller and colleagues summarized the evidence derived from neuroimaging experiments (using fMRI or PET) of group comparisons between adults with unipolar depression and healthy controls and reporting significant activation differences between patients and controls during emotional or cognitive tasks [60]. Several inconsistencies across available studies emerged from this systematic review. Brain metabolism in MDD was also specifically addressed in several voxel-based PET studies which were submitted to a meta-analytical approach by Su et al. [61]. Again while decreased prefrontal, insular, and limbic cerebral glucose metabolism was repeatedly highlighted in depressed patients with respect to healthy controls, available literature has not always been consistent. In this framework, the involvement of specific regions such as insula, limbic system, basal ganglia, thalamus, and cerebellum was more frequently reported, suggesting that these regions are likely to play a key role in the pathophysiology of depression. In keeping with these results, convergent change in the limbic-cortical brain circuit in depression compared to controls was also found in multi-modal imaging studies involving both PET and MRI data. Reported 
specific changes include lower gray matter volumes in amygdala, dorsal frontomedian cortex, and the right paracingulate cortex, as well as relative hypermetabolism in the right subgenual and pregenual anterior cingulate cortices. Building a strong and evidence-based mapping of these alterations in MDD is relevant as these regions could serve as a specific region-of-interest-fordisease template for both in vivo imaging in individual patients and postmortem histopathologic exploration [62]. Other PET studies investigated altered function related to different pathways including 5-HT receptor and transporter dysfunction in neuropsychiatric disorders. Indeed, impairment of serotonin receptor and transporter function is increasingly recognized to play a major role in the pathophysiology of neuropsychiatric diseases including anxiety disorder, major depressive disorder, bipolar disorder, and schizophrenia. In particular, a retrospective analysis revealed that these psychiatric disorders differed in affected brain regions, affected synaptic constituents as well as direction of dysfunction in terms of either sensitization or desensitization of transporter and receptor binding sites [63].

Striatal presynaptic dopamine pathway has been the most frequent target for PET and SPECT imaging in schizophrenia [64]. As a matter of fact, the role of striatal dopaminergic neurotransmission in the onset of symptoms of schizophrenia (including psychotic symptoms) is currently targeted by dopaminergic drugs. A specific marker of the integrity of presynaptic dopamine neurons in the striatum, the density of striatal dopamine terminals, can be quantified through molecular neuroimaging of DAT. A meta-analysis of DAT density in the striatum of schizophrenic patients demonstrated that striatal DAT density was not significantly different between patients and controls [64]. Similar negative findings were regionally confirmed in putamen and caudate. There was no moderating effect for external factors such as antipsychotic medication or illness duration. Accordingly, the authors concluded that loss of integrity of striatal dopaminergic synapses is not critical for the emergence of schizophrenia and these findings are relevant for further refining dopaminergic hypotheses of schizophrenia (with possible repercussion on interventional studies aiming to identify new treatment options). By contrast, a different window on presynaptic dopaminergic function was opened by studies exploring this pathway through ${ }^{11} \mathrm{C}-/{ }^{18} \mathrm{~F}$-DOPA PET [65]. Available studies were summarized in the meta-analysis by Fusar-Poli and MeyerLindenberg [65]: patients with schizophrenia showed increased striatal uptake as compared with controls and this finding was regionally confirmed in both caudate and putamen. Finally, no significant effect of age, illness duration, gender, psychotic symptoms, and exposure to antipsychotics was highlighted. Of note, sensitivity analysis confirmed robustness of meta-analytic findings. Finally, data from animal models and from postmortem studies suggest that schizophrenia is associated with brain GABAergic dysfunction. However, it is still unclear the extent of this effect in vivo studies of GABA function in patients with schizophrenia [66].

PET and SPECT studies with several tracers have been used on other neuropsychiatric diseases including obsessive compulsive disorders, Tourette's syndrome (TS), and attention deficit hyperactivity disorder (ADHD) as well as to test brain function in specific conditions such as in case of ecstasy/polydrug use and disorders of consciousness. In particular, dopaminergic imaging with PET and SPECT was able to demonstrate dopaminergic alterations in TS and pathophysiology and psychostimulant treatment of attention deficit in ADHD. In fact, dopaminergic alterations in TS are in line with the current pathophysiological hypotheses of a tonic-phasic dysfunction in the dopamine system although available studies are characterized by low effect sizes due to the heterogeneity of the disease [67]. Similarly, although dopaminergic studies in ADHD yielded inconsistent results often demonstrating either high and low striatal dopamine transporter levels across different studies, a systematic review of the available evidence highlighted that striatal dopamine transporter density in ADHD is depended on previous psychostimulant exposure, with lower density in drug-naive subjects and higher density in previously medicated patients [68]. More general approaches on brain perfusion and metabolism by means of SPECT and PET allowed to demonstrate that 
pharmacological and psychological treatments reduce resting cortico-striato-thalamo-cortical circuit activity in obsessive compulsive disorder. Similarly markedly reduced activity within midline cortical and subcortical sites (anatomical structures linked to the default-mode network) are present in patients with disorders of consciousness $[69,70]$. Finally, PET studies on postsynaptic 5HT2A receptor imaging allowed to highlight that serotonin axons with the longest projections from the raphe nuclei might be more markedly affected by ecstasy/MDMA use.

\subsection{Conclusions}

In conclusion, a huge body of literature has highlighted an ongoing and promising role of PET with different tracers in neurodegenerative dementia, movement and psychiatric disorders. In some clinical settings such as in case patients with mild cognitive impairment and dementia, for the differential diagnosis of underlying etiology as well as for the early and accurate identification of patients with neurodegenerative parkinsonian syndromes, PET has gained an increasing relevant clinical role. By contrast the possibility to accurately quantify neurotransmission with different tracers is increasingly supporting the use of PET technology for pathophysiological and interventional studies in patients with psychiatric disorders. In both cases (clinical use of PET in neurodegenerative dementia and parkinsonian syndromes and research use of PET in psychiatric disorders), it is crucial to proceed with robust methodology which starts with the systematic evaluation of evidence-based results of previous studies. Only this approach will allow to balance costs and clinical advancement, thus meeting the needs of both patients and health-care systems.

\section{References}

1. Albert MS, DeKosky ST, Dickson D, et al. The diagnosis of mild cognitive impairment due to Alzheimer's disease: recommendations from the National Institute on Aging and Alzheimer's Association workgroup. Alzheimers Dement. 2011;7:270-9.
2. Dubois B, Feldman HH, Jacova C, et al. Advancing research diagnostic criteria for Alzheimer's disease: the IWG-2 criteria. Lancet Neurol. 2014;13:614-29.

3. Nasrallah IM, Wolk DA. Multimodality imaging of Alzheimer disease and other neurodegenerative dementias. J Nucl Med. 2014;55:2003-11.

4. Boccardi M, Nicolosi V, Festari C, Bianchetti A, Cappa S, Chiasserini D, et al. Italian consensus recommendations for a biomarker-based aetiological diagnosis in mild cognitive impairment patients. Eur J Neurol. 2019;27(3):475-83. https://doi.org/10.1111/ ene.14117.

5. Frisoni GB, Boccardi M, Barkhof F, Blennow K, Cappa S, Chiotis K, et al. Strategic roadmap for an early diagnosis of Alzheimer's disease based on biomarkers. Lancet Neurol. 2017;16(8):661-76.

6. Narayanaswami V, Dahl K, Bernard-Gauthier V, Josephson L, Cumming P, Vasdev N. Emerging PET radiotracers and targets for imaging of neuroinflammation in neurodegenerative diseases: outlook beyond TSPO. Mol Imaging. 2018;17:1536012118792317.

7. Chen X, Li M, Wang S, Zhu H, Xiong Y, Liu $\mathrm{X}$. Pittsburgh compound B retention and progression of cognitive status - a meta-analysis. Eur J Neurol. 2014;21(8):1060-7.

8. Kovacs GG. Molecular pathology of neurodegenerative diseases: principles and practice. J Clin Pathol. 2019;72(11):725-35.

9. Seo EH, Park WY, Choo IH. Structural MRI and amyloid PET imaging for prediction of conversion to Alzheimer's disease in patients with mild cognitive impairment: a meta-analysis. Psychiatry Investig. 2017;14(2):205-15.

10. Niccolini F, Pagano G, Fusar-Poli P, Wood A, Mrzljak L, Sampaio C, et al. Striatal molecular alterations in HD gene carriers: a systematic review and meta-analysis of PET studies. J Neurol Neurosurg Psychiatry. 2018;89(2):185-96.

11. Smailagic N, Lafortune L, Kelly S, Hyde C, Brayne C. 18F-FDG PET for prediction of conversion to Alzheimer's disease dementia in people with mild cognitive impairment: an updated systematic review of test accuracy. J Alzheimers Dis. 2018;64(4):1175-94.

12. Morbelli S, Garibotto V, Van De Giessen E, Arbizu J, Chételat G, Drezgza A, et al. European Association of Nuclear Medicine. A Cochrane review on brain $\left[{ }^{18} \mathrm{~F}\right] \mathrm{FDG}$ PET in dementia: limitations and future perspectives. Eur J Nucl Med Mol Imaging. 2015;42(10):1487-91.

13. Dukart J, Mueller K, Barthel H, Villringer A, Sabri O, Schroeter ML, Alzheimer's Disease Neuroimaging Initiative. Meta-analysis based SVM classification enables accurate detection of Alzheimer's disease across different clinical centers using FDG-PET and MRI. Psychiatry Res. 2012;212(3):230-6.

14. Morbelli S, Bauckneht M. Amyloid PET imaging: standardization and integration with other Alzheimer's disease biomarkers. Methods Mol Biol. 1750;2018:203-12. 
15. Zhang S, Han D, Tan X, Feng J, Guo Y, Ding Y. Diagnostic accuracy of 18 F-FDG and 11 C-PIBPET for prediction of short-term conversion to Alzheimer's disease in subjects with mild cognitive impairment. Int J Clin Pract. 2012;66(2):185-98.

16. He W, Liu D, Radua J, Li G, Han B, Sun Z. Metaanalytic comparison between PIB-PET and FDG-PET results in Alzheimer's disease and MCI. Cell Biochem Biophys. 2015;71(1):17-26.

17. Morbelli S, Bauckneht M, Scheltens P. Imaging biomarkers in Alzheimer's disease: added value in the clinical setting. Q J Nucl Med Mol Imaging. 2017;61(4):360-71.

18. Morris E, Chalkidou A, Hammers A, Peacock J, Summers J, Keevil S. Diagnostic accuracy of (18)F amyloid PET tracers for the diagnosis of Alzheimer's disease: a systematic review and meta-analysis. Eur J Nucl Med Mol Imaging. 2016;43(2):374-85.

19. Shea YF, Barker W, Greig-Gusto MT, Loewenstein DA, Duara R, DeKosky ST. Impact of amyloid PET imaging in the memory clinic: a systematic review and meta-analysis. J Alzheimers Dis. 2018;64(1):323-35.

20. Bisenius S, Neumann J, Schroeter ML. Validating new diagnostic imaging criteria for primary progressive aphasia via anatomical likelihood estimation meta-analyses. Eur J Neurol. 2016;23(4):704-12.

21. Bergeron D, Gorno-Tempini ML, Rabinovici GD, Santos-Santos MA, Seeley W, Miller BL, et al. Prevalence of amyloid- $\beta$ pathology in distinct variants of primary progressive aphasia. Ann Neurol. 2018;84(5):729-40.

22. Baker JE, Lim YY, Pietrzak RH, Hassenstab J, Snyder PJ, Masters CL, et al. Cognitive impairment and decline in cognitively normal older adults with high amyloid- $\beta$ : a meta-analysis. Alzheimers Dement. 2017;6:108-21.

23. Hedden T, Oh H, Younger AP, Patel TA. Meta-analysis of amyloid-cognition relations in cognitively normal older adults. Neurology. 2013;80(14):1341-8.

24. Charidimou A, Farid K, Tsai HH, Tsai LK, Yen RF, Baron JC. Amyloid-PET burden and regional distribution in cerebral amyloid angiopathy: a systematic review and meta-analysis of biomarker performance. J Neurol Neurosurg Psychiatry. 2018;89(4):410-7.

25. Charidimou A, Farid K, Baron JC. AmyloidPET in sporadic cerebral amyloid angiopathy: a diagnostic accuracy meta-analysis. Neurology. 2017;89(14):1490-8.

26. Martínez G, Vernooij RW, Fuentes Padilla P, Zamora J, Bonfill Cosp X, Flicker L. 18F PET with florbetapir for the early diagnosis of Alzheimer's disease dementia and other dementias in people with mild cognitive impairment (MCI). Cochrane Database Syst Rev. 2017;11:CD012216.

27. Fahn S. Classification of movement disorders. Mov Disord. 2011;26(6):947-57.

28. Jankovic J. Parkinson's disease: clinical features and diagnosis. J Neurol Neurosurg Psychiatry. 2008;79(4):368-76.
29. Braak H, Del Tredici K, Rüb U, de Vos RA, Jansen Steur EN, Braak E. Staging of brain pathology related to sporadic Parkinson's disease. Neurobiol Aging. 2003;24(2):197-211.

30. Kalia LV, Lang AE. Parkinson's disease. Lancet. 2015;386(9996):896-912.

31. Politis M, Wu K, Molloy S, Bain PG, Chaudhuri KR, Piccini P. Parkinson's disease symptoms: the patient's perspective. Mov Disord. 2010;25(11):1646-51.

32. Garnett ES, Firnau G, Nahmias C. Dopamine visualized in the basal ganglia of living man. Nature. 1983;305(5930):137-8.

33. Meiser J, Weindl D, Hiller K. Complexity of dopamine metabolism. Cell Commun Signal. 2013;11(1):34.

34. Uhl GR. Dopamine transporter: basic science and human variation of a key molecule for dopaminergic function, locomotion, and parkinsonism. Mov Disord. 2003;18(Suppl 7):S71-80.

35. Karrer TM, Josef AK, Mata R, Morris ED, SamanezLarkin GR. Reduced dopamine receptors and transporters but not synthesis capacity in normal aging adults: a meta-analysis. Neurobiol Aging. 2017;57:36-46.

36. Kaasinen V, Vahlberg T. Striatal dopamine in Parkinson disease: a meta-analysis of imaging studies. Ann Neurol. 2017;82(6):873-82.

37. Lee CS, Samii A, Sossi V, Ruth TJ, Schulzer M, Holden JE, et al. In vivo positron emission tomographic evidence for compensatory changes in presynaptic dopaminergic nerve terminals in Parkinson's disease. Ann Neurol. 2000;47(4):493-503.

38. Houle S, Ginovart N, Hussey D, Meyer JH, Wilson AA. Imaging the serotonin transporter with positron emission tomography: initial human studies with [11C]DAPP and [11C]DASB. Eur J Nucl Med. 2000;27(11):1719-22.

39. Pagano G, Niccolini F, Fusar-Poli P, Politis M. Serotonin transporter in Parkinson's disease: a meta-analysis of positron emission tomography studies. Ann Neurol. 2017;81(2):171-80.

40. Rizzo G, Copetti M, Arcuti S, Martino D, Fontana A, Logroscino G. Accuracy of clinical diagnosis of Parkinson disease: a systematic review and metaanalysis. Neurology. 2016;86(6):566-76.

41. Berg D, Postuma RB, Adler CH, Bloem BR, Chan P, Dubois B, et al. MDS research criteria for prodromal Parkinson's disease. Mov Disord. 2015;30(12):1600-11.

42. Iranzo A, Santamaría J, Valldeoriola F, Serradell M, Salamero M, Gaig C, et al. Dopamine transporter imaging deficit predicts early transition to synucleinopathy in idiopathic rapid eye movement sleep behavior disorder. Ann Neurol. 2017;82(3):419-28.

43. Bauckneht M, Chincarini A, De Carli F, Terzaghi M, Morbelli S, Nobili F, et al. Presynaptic dopaminergic neuroimaging in REM sleep behavior disorder: a systematic review and meta-analysis. Sleep Med Rev. 2017;41:266-74. 
44. Benamer TS, Patterson J, Grosset DG, Booij J, de Bruin K, van Royen E, et al. Accurate differentiation of parkinsonism and essential tremor using visual assessment of [123I]-FP-CIT SPECT imaging: the [123I]-FP-CIT study group. Mov Disord. 2000;15(3):503-10.

45. Tinazzi M, Antonini A, Bovi T, Pasquin I, Steinmayr M, Moretto G, et al. Clinical and [123I]FP-CIT SPET imaging follow-up in patients with drug-induced parkinsonism. J Neurol. 2009;256(6):910-5.

46. Nicastro N, Garibotto V, Badoud S, Burkhard PR. Scan without evidence of dopaminergic deficit: a 10-year retrospective study. Parkinsonism Relat Disord. 2016;31:53-8.

47. Brücke T, Asenbaum S, Pirker W, Djamshidian S, Wenger S, Wöber C, et al. Measurement of the dopaminergic degeneration in Parkinson's disease with [123I] beta-CIT and SPECT. Correlation with clinical findings and comparison with multiple system atrophy and progressive supranuclear palsy. J Neural Transm Suppl. 1997;50:9-24.

48. Albrecht F, Ballarini T, Neumann J, Schroeter ML. FDG-PET hypometabolism is more sensitive than MRI atrophy in Parkinson's disease: a wholebrain multimodal imaging meta-analysis. Neuroimage Clin. 2019;21:101594.

49. D’Antona R, Baron JC, Samson Y, Serdaru M, Viader F, Agid Y, et al. Subcortical dementia. Frontal cortex hypometabolism detected by positron tomography in patients with progressive supranuclear palsy. Brain. 1985;108(Pt 3):785-99.

50. Eidelberg D, Dhawan V, Moeller JR, Sidtis JJ, Ginos JZ, Strother SC, et al. The metabolic landscape of cortico-basal ganglionic degeneration: regional asymmetries studied with positron emission tomography. J Neurol Neurosurg Psychiatry. 1991;54(10):856-62.

51. Meyer PT, Frings L, Rücker G, Hellwig S. (18) F-FDG PET in parkinsonism: differential diagnosis and evaluation of cognitive impairment. J Nucl Med. 2017;58(12):1888-98.

52. McKeith IG, Boeve BF, Dickson DW, Halliday G, Taylor JP, Weintraub D, et al. Diagnosis and management of dementia with Lewy bodies: fourth consensus report of the DLB Consortium. Neurology. 2017;89(1):88-100.

53. Graff-Radford J, Murray ME, Lowe VJ, Boeve BF, Ferman TJ, Przybelski SA, et al. Dementia with Lewy bodies: basis of cingulate island sign. Neurology. 2014;83(9):801-9.

54. Tang CC, Feigin A, Ma Y, Habeck C, Paulsen JS, Leenders KL, et al. Metabolic network as a progression biomarker of premanifest Huntington's disease. J Clin Invest. 2013;123(9):4076-88.

55. Fowler JS, Volkow ND, Logan J, Wang GJ, MacGregor RR, Schyler D, et al. Slow recovery of human brain MAO B after L-deprenyl (Selegeline) withdrawal. Synapse. 1994;18(2):86-93.

56. Marek K, Jennings D, Seibyl J. Imaging the dopamine system to assess disease-modifying drugs: studies comparing dopamine agonists and levodopa. Neurology. 2003;61(6 Suppl 3):S43-8.

57. Brooks DJ, Ibanez V, Sawle GV, Playford ED, Quinn $\mathrm{N}$, Mathias CJ, et al. Striatal D2 receptor status in patients with Parkinson's disease, striatonigral degeneration, and progressive supranuclear palsy, measured with 11C-raclopride and positron emission tomography. Ann Neurol. 1992;31(2):184-92.

58. Martini A, Dal Lago D, Edelstyn NMJ, Salgarello M, Lugoboni F, Tamburin S. Dopaminergic neurotransmission in patients with Parkinson's disease and impulse control disorders: a systematic review and meta-analysis of PET and SPECT studies. Front Neurol. 2018;9:1018.

59. Nobili F, Arbizu J, Bouwman F, Drzezga A, Agosta F, Nestor P, et al. EANM-EAN Task Force for the Prescription of FDG-PET for Dementing Neurodegenerative Disorders. European Association of Nuclear Medicine and European Academy of Neurology recommendations for the use of brain (18) F-fluorodeoxyglucose positron emission tomography in neurodegenerative cognitive impairment and dementia: Delphi consensus. Eur J Neurol. 2018;25(10):1201-17.

60. Müller VI, Cieslik EC, Serbanescu I, Laird AR, Fox PT, Eickhoff SB. Altered brain activity in unipolar depression revisited: meta-analyses of neuroimaging studies. JAMA Psychiat. 2017;74(1):47-55.

61. Su L, Cai Y, Xu Y, Dutt A, Shi S, Bramon E. Cerebral metabolism in major depressive disorder: a voxelbased meta-analysis of positron emission tomography studies. BMC Psychiatry. 2014;14:321.

62. Sacher J, Neumann J, Fünfstück T, Soliman A, Villringer A, Schroeter ML. Mapping the depressed brain: a meta-analysis of structural and functional alterations in major depressive disorder. J Affect Disord. 2012;140(2):142-8.

63. Nikolaus S, Müller HW, Hautzel H. Different patterns of 5-HT receptor and transporter dysfunction in neuropsychiatric disorders-a comparative analysis of in vivo imaging findings. Rev Neurosci. 2016;27(1):27-59.

64. Fusar-Poli P, Meyer-Lindenberg A. Striatal presynaptic dopamine in schizophrenia, part I: meta-analysis of dopamine active transporter (DAT) density. Schizophr Bull. 2012;39(1):22-32.

65. Fusar-Poli P, Meyer-Lindenberg A. Striatal presynaptic dopamine in schizophrenia, part II: meta-analysis of [(18)F/(11)C]-DOPA PET studies. Schizophr Bull. 2013;39(1):33-42.

66. Egerton A, Modinos G, Ferrera D, McGuire P. Neuroimaging studies of GABA in schizophrenia: a systematic review with meta-analysis. Transl Psychiatry. 2017;7(6):e1147.

67. Hienert M, Gryglewski G, Stamenkovic M, Kasper S, Lanzenberger R. Striatal dopaminergic alterations in Tourette's syndrome: a meta-analysis based on 16 PET and SPECT neuroimaging studies. Transl Psychiatry. 2018;8(1):143. 
68. Fusar-Poli P, Rubia K, Rossi G, Sartori G, Balottin U. Striatal dopamine transporter alterations in ADHD: pathophysiology or adaptation to psychostimulants? A meta-analysis. Am J Psychiatry. 2012;169(3):264-72.

69. Van der Straten AL, Denys D, van Wingen GA. Impact of treatment on resting cerebral blood flow and metabolism in obsessive compulsive disorder: a metaanalysis. Sci Rep. 2017;7(1): 17464.

70. Hannawi Y, Lindquist MA, Caffo BS, Sair HI, Stevens RD. Resting brain activity in disorders of consciousness: a systematic review and meta-analysis. Neurology. 2017;84(12):1272-80.

Open Access This chapter is licensed under the terms of the Creative Commons Attribution 4.0 International License (http://creativecommons.org/licenses/by/4.0/), which permits use, sharing, adaptation, distribution and reproduction in any medium or format, as long as you give appropriate credit to the original author(s) and the source, provide a link to the Creative Commons license and indicate if changes were made.

The images or other third party material in this chapter are included in the chapter's Creative Commons license, unless indicated otherwise in a credit line to the material. If material is not included in the chapter's Creative Commons license and your intended use is not permitted by statutory regulation or exceeds the permitted use, you will need to obtain permission directly from the copyright holder. 